\title{
Fines, L. (2016). Les crimes environnementaux et l'innocence persécutrice. Paris: L'Harmattan, 175 p.
}

\author{
Jeanne Simard ${ }^{a}$, Marc-André Morency ${ }^{b}$
}

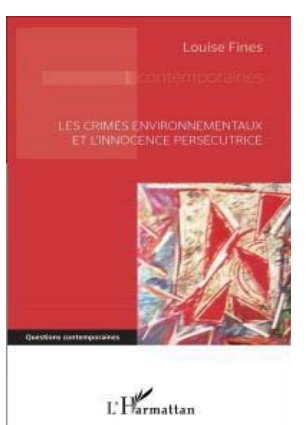

L'auteure, criminologue, a publié de nombreux ouvrages et articles sur la criminalité en col blanc (Les crimes invisibles. Délits contemporains, dénonciation et temps de réaction [2013]; Négociations et crimes en col blanc. Immunités réciproques [2013]; Irresponsabilités récurrentes des élites. Accidents fortuits et crimes en col blanc [2015]; Entre sphères légales et réseaux illégaux. Le jeu de la collusion [2015]). Cet ouvrage, publié en 2016, explore une branche plus fine de la criminalité en col blanc, celle qui a pour objet les crimes contre l'environnement et les infractions du même ordre ayant affecté la santé ou la sécurité des travailleurs.

Les conditions de la croissance économique dans un contexte de mondialisation des affaires et de la recherche du profit à court terme ont amené les entreprises et leurs dirigeants à délocaliser de nombreuses pratiques désastreuses pour l'environnement et la planète. Pas une semaine ne se passe sans que les médias ne rapportent les signes patents de cette dérive généralisée à l'échelle du globe. Les dommages sont immenses, avec des conséquences souvent dramatiques et durables pour les victimes actuelles (la population en général et les travailleurs au sein des entreprises) et les générations futures. Que ces dommages soient le produit d'actes délibérés ou le résultat de négligences, les poursuites devant les tribunaux sont toujours rares et les condamnations encore plus quoique, depuis 2016, la Cour pénale internationale est en mesure de traiter des affaires où la destruction de l'environnement se trouve liée à des violations des droits de l'homme.

Dans la foulée de la crise économique, financière et sociale déclenchée en 2007-2008 et le mouvement global de protestation de la société civile en faveur du droit à un habitat viable et de la protection de la biodiversité, les recherches en matière de protection de l'environnement et des crimes à cet égard, se sont multipliées. Cependant, les études permettant d'aborder ces problématiques sont souvent fort techniques, produites en silos disciplinaires, s'adressant presque exclusivement aux initiés. Cet ouvrage de Louise Fines se propose de présenter, dans une perspective systémique, les aspects institutionnels (juridique, politique) et psychosociaux de cette problématique afin que les criminalistes, mais aussi un lectorat varié (juristes, gestionnaires, dirigeants d'entreprise, fonctionnaires, journalistes, observateurs) puissent mieux comprendre l'ampleur et les raisons de ces dérives et la difficulté de prévenir, de réprimer et de punir, par les voies du système judiciaire traditionnel, les conduites à l'origine de ces désordres. Comme le résume bien l'auteure, il y a tout lieu « de revoir l'usage de la terminologie de la catastrophe industrielle, pour y inclure, outre les conduites des entreprises qui œuvrent en première ligne [...], les actions de toutes les sociétés commerciales, institutions, banques, administrations politiques... qui contribuent par leur silence, leurs réactions tardives et leurs réponses différées, à la création de conditions favorables aux activités illégales de délinquants récidivistes? » (p. 10). Dit autrement, « est-ce que la position structurelle des accusés est de nature à intervenir dans la manière dont les dispositions sont prises en vue de prévenir les crimes commis contre l'environnement, dans la façon d'interpréter les conduites des accusés (individus, organisations), de mener les enquêtes et par voie de conséquence, dans la manière de réagir aux actions en cours, d'y mettre un terme, ou bien de les tolérer? » (p. 18). Par position structurelle des accusés, l'auteure entend le pouvoir afférent à leur position dans une hiérarchie sociale inégalitaire qu'ils contribuent à maintenir.

\footnotetext{
a Professeure, LL. D., avocate, F. Adm.A., Université du Québec à Chicoutimi

b Professeur retraité, sociologue, Université du Québec à Chicoutimi
} 
Le fil conducteur ou la posture épistémologique des travaux de l'auteure dans cette étude des crimes environnementaux est celui du concept d'« innocence persécutrice » élaboré au cours des années 1930 par l'Autrichien Karl Krauss (1874-1936), un fin observateur de la montée de l'idéologie qui allait présider à la Seconde Guerre mondiale et aux atrocités commises sous son égide. L'« innocence persécutrice » rend compte de la capacité des agresseurs à rationaliser, «à justifier leurs actions illégales sous couvert d'une prétention à la légitime défense » (p. 20). Krauss observait que « ceux-là mêmes qui tuent ou qui ont tué rejettent la faute sur des victimes qui n'ont joué parfois qu'un rôle mineur ou secondaire dans les événements en question, une façon en quelque sorte de détourner l'attention de leurs propres crimes et de désigner [persécuter] les "vrais" coupables » (p. 157). Sous le voile de «l'innocence », la vérité est alors trafiquée, fabriquée sous forme de définitions de situation institutionnalisées dans les rapports politiques et juridiques ou le cas échéant, corporatifs. Cet ensemble institutionnel devient un système doué de vertus d'autofécondation. Appliqué aux crimes environnementaux, ce concept permet à l'auteure d'apporter une lecture multidimensionnelle des événements et non plus simplement juridiques, en quel cas, le fauteur de troubles trouve à excuser ses comportements les plus odieux, bien illustrés dans la foulée des fameuses « lois de Nuremberg ». À notre époque, où les fausses nouvelles (fake news) abondent dans les réseaux sociaux, ces réflexions de Karl Krauss paraissent bien trouver à nouveau toute leur actualité.

L'intérêt de l'ouvrage est de montrer en détail l'opérationnalisation sur le plan de la criminalité environnementale de ce concept de «l'innocence persécutrice», au moyen de quatre cas des plus intéressants tirés de l'actualité internationale contemporaine : 1) la contamination des sols et d'une communauté par des produits toxiques rejetés pendant des années par la société Hooker Chemical Company dans le Love Canal (Niagara Falls, État de New York); 2) l'explosion en 2010 de la mine de charbon Upper Big Branch à Montcoal en VirginieOccidentale; 3) la contamination parasitaire de l'eau potable en 2010 de la ville d'Östersund en Suède; 4) la pollution d'un cours d'eau et du bassin d'Arcachon après la rupture en 2012 d'une cuve de la papeterie Smurfit Kappa en France. À partir de rapports gouvernementaux et organisationnels, d'archives judiciaires, d'articles de journaux et de diverses références médiatiques, l'auteure a réalisé un travail minutieux de recherche et d'analyse afin de mettre au jour la manière dont la position structurelle ou le pouvoir politique des infracteurs dans ces quatre affaires a été susceptible de jouer un rôle dans l'émergence, la négociation et la durée (p. ex., prolonger grâce à des ressources corporatives les procédures judiciaires) de ces crimes et comment les discours politiques et économiques ont pu les justifier. Dans sa démonstration, l'auteure rappelle les accusations portées contre les infracteurs présumés ainsi que les verdicts prononcés contre eux. Elle recense les diverses stratégies de défense élaborées par ces derniers en vue de justifier leurs actions tout en s'intéressant aux efforts déployés par les victimes auprès du système judiciaire pour faire reconnaitre leurs droits. Elle décrit les dérives des instances de surveillance dans ces affaires, dont les agences de régulation, ainsi que la capacité des infracteurs présumés à exploiter la dimension temporelle à leur avantage, et leurs manœuvres (dont le contrôle de l'information) en vue d'occulter leurs crimes. Elle discute ensuite du concept de l'innocence persécutrice à l'aune du pouvoir politique ou de la position structurelle privilégiée des acteurs et de l'effet amplificateur de la diffusion de la responsabilité des acteurs à mesure que le temps passe. Elle analyse tout particulièrement les discours déployés à grande échelle par ceux qui tentent de justifier les crimes environnementaux.

En synthèse, l'ouvrage Les crimes environnementaux et l'innocence persécutrice ajoute une pièce fort utile à la réflexion sur les conditions systémiques favorisant l'émergence et la durée de crimes environnementaux et, par ricochet, les situations venant compromettre la santé et la sécurité des travailleurs, des communautés et, à la limite, celle des générations futures. Cette réflexion sur les crimes environnementaux est courageuse, car elle demande d'aborder des situations complexes où s'affrontent les contradictions sociales, les jeux et les discours politiques et économiques, les intérêts divergents; elle examine le rôle des institutions et la prise de décision éthique alors que de nombreux ordres sociaux en cause (droit international, droit national, déontologie, morale, règlements de régie interne des organisations, etc.) interagissent, voire se contredisent. C'est une lecture d'un grand intérêt pour le public et un apport sans conteste aux disciplines visant les crimes en col blanc. 